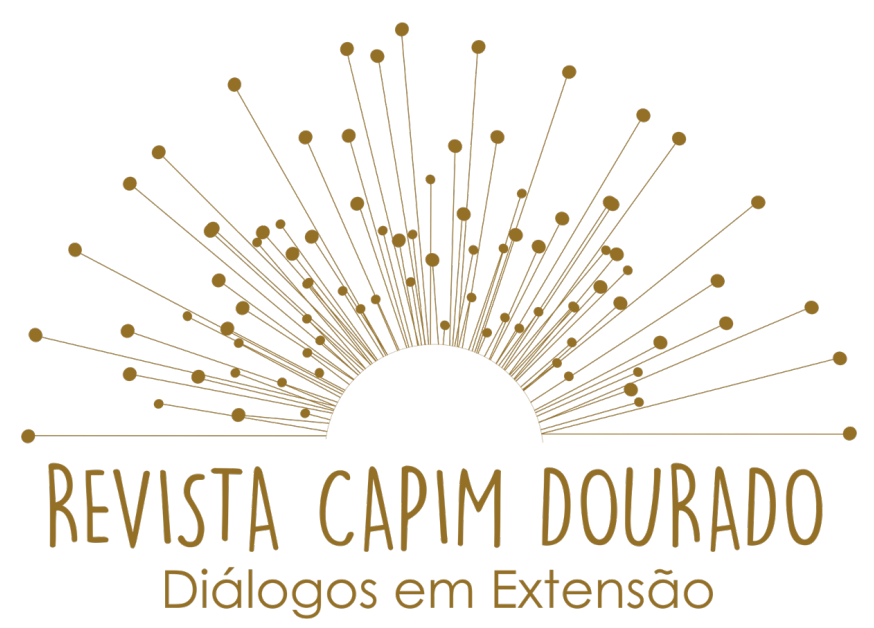

ISSN n² 2595-7341

Vol. 3, n. 1, Janeiro-Abril, 2020

DOI: http://dx.doi.org/10.20873/uft.2595-7341.2020v3n1p41

\title{
PROMOÇÃO DE PRÁTICAS ALIMENTARES SAUDÁVEIS PARA IDOSOS MATRICULADOS NA UNIVERSIDADE DA MATURIDADE
}

\section{PROMOTION OF HEALTHY FOOD PRACTICES FOR ELDERLY ENROLLED AT THE MATURITY UNIVERSITY}

PROMOCIÓN DE PRÁCTICAS ALIMENTARIAS SALUDABLES PARA MAYORES INSCRITOS EN LA UNIVERSIDAD DE MADUREZ

\section{Samara Vanderlley Costa Matos ${ }^{1}$ \\ Isabela Cêa ${ }^{2}$ \\ Luiz Sinésio Silva Neto ${ }^{3}$ \\ Daniella Pires Nunes ${ }^{4}$ \\ Fabiane Aparecida Canaan Rezende Rezende ${ }^{5}$}

\section{RESUMO}

Sabe se que a população idosa vem crescendo de forma significativa, seguindo a tendência mundial de alargamento do topo da pirâmide. Tendo em vista esse

\footnotetext{
${ }^{1}$ Universidade Federal do Tocantins (UFT), Curso de Nutrição. Bolsista PIBEX Acadêmico. E-mail: samaravcmatos@hotmail.com.

2 Universidade Federal do Tocantins (UFT), Curso de Nutrição. Bolsista PIBEX Acadêmico. E-mail: Bela.Cea@hotmail.com.

3 Universidade Federal do Tocantins (UFT), Curso de Medicina. Docente. E-mail: luizneto@mail.uft.edu.br.

${ }^{4}$ NUNES, Daniella Pires. Universidade Federal do Tocantins (UFT), Curso de Enfermagem. Docente. E-mail: daniellanunes@mail.uft.edu.br.

5 Universidade Federal do Tocantins (UFT), Curso de Nutrição. Docente. E-mail: facrezende@uft.edu.br.
} 


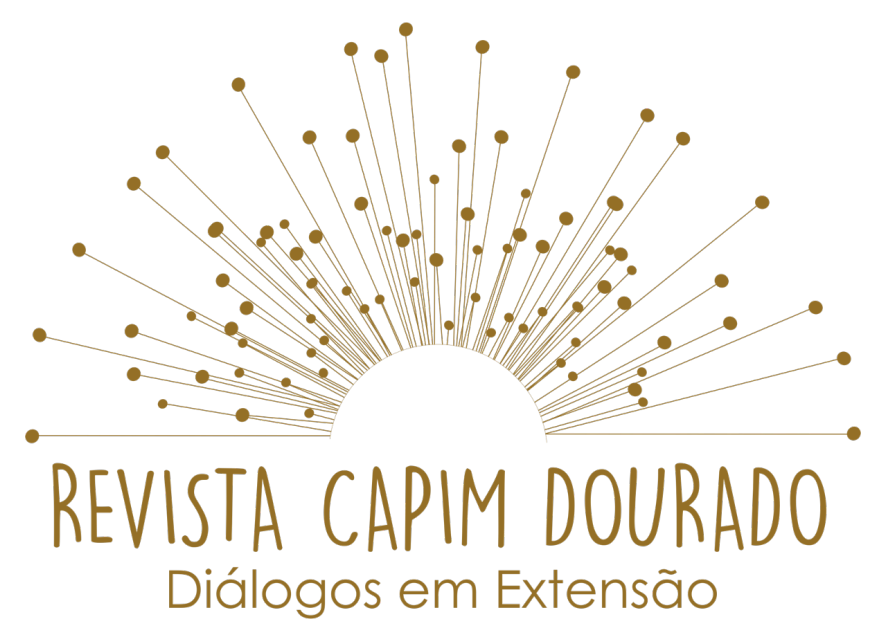

ISSN n² 2595-7341

Vol. 3, n. 1, Janeiro-Abril, 2020

DOI: http://dx.doi.org/10.20873/uft.2595-7341.2020v3n1p41

envelhecimento da população buscar lo de uma forma saudável e minimizar impactos que o avançar da idade pode trazer são propósitos de idosos que buscam a qualidade de vida. O objetivo deste trabalho foi promover ações de educação alimentar e nutricional (EAN), levando ao conhecimento e empoderamento no que se refere a alimentação, com questões críticas e reflexivas. Os procedimentos metodológicos utilizados foram atendimento gerontológico através da Avaliação Geriátrica Ampla (AGA), a partir das respostas obtidas com a AGA elaborava se um plano de cuidados individualizado, e ações de educação alimentar e nutricional através de palestras e oficinas. Os resultados encontrados foram de multimorbidade, alto índice de doenças articulares e osteoporose, além da Hipertensão Arterial, o que nos leva a observar a importância da educação alimentar e nutricional na contribuição de evitar o aceleramento de comobidades já existentes e no tratamento de carências nutricionais além de promover a autonomia alimentar dessa população.

PALAVRAS-CHAVE: Envelhecimento. Promoção da saúde. Aconselhamento nutricional. Alimentação saudável.

\section{ABSTRACT}

It is known that the elderly population has been growing significantly, following the global trend of widening the top of the pyramid. In view of this aging population, seeking it in a healthy way and minimizing impacts that advancing age can bring are the purposes of the elderly who seek quality of life. The objective of this work was to promote actions of food and nutrition education (EAN), leading to knowledge and empowerment regarding food, with critical and reflective questions. The methodological procedures used were gerontological care through the Comprehensive Geriatric Assessment (AGA), based on the answers obtained with the AGA, an individualized care plan was elaborated, and food and nutrition education actions through lectures and workshops. The results found were of multimorbidity, high rate of joint diseases and osteoporosis, in 


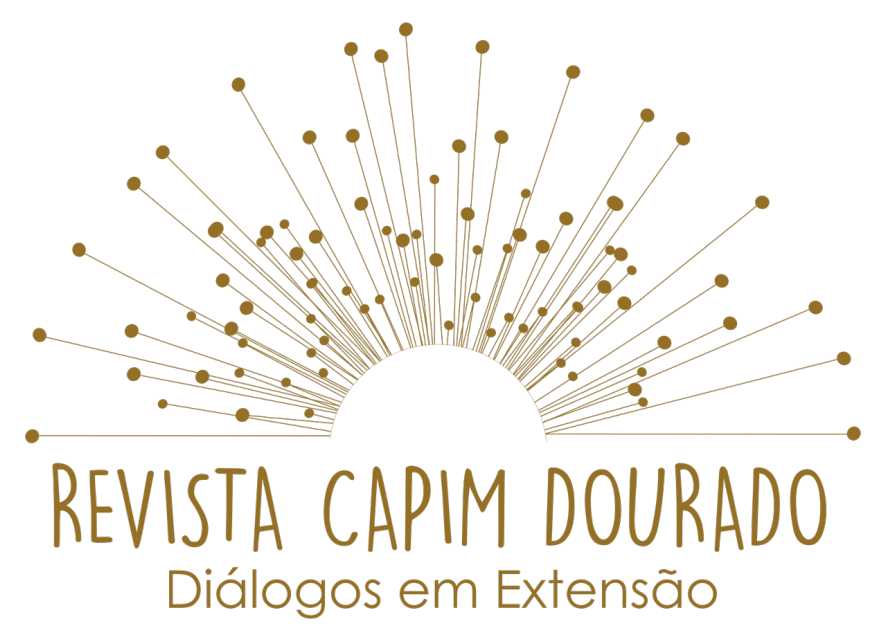

ISSN n² 2595-7341

Vol. 3, n. 1, Janeiro-Abril, 2020

DOI: http://dx.doi.org/10.20873/uft.2595-7341.2020v3n1p41

addition to Arterial Hypertension, which leads us to observe the importance of food and nutrition education in the contribution to avoid the acceleration of existing comorbidities and in the treatment of nutritional deficiencies beyond promote the food autonomy of this population.

KEYWORDS: Aging. Health promotion. Nutritional advice. Healthy eating.

\section{RESUMEN}

Se sabe que la población anciana ha ido creciendo de manera significativa, siguiendo la tendencia global de ensanchar la cúspide de la pirámide. Ante este envejecimiento de la población, buscarla de manera saludable y minimizar los impactos que puede traer la edad avanzada son propósitos de las personas mayores que buscan calidad de vida. El objetivo de este trabajo fue promover acciones de educación alimentaria y nutricional (EAN), conducentes al conocimiento y empoderamiento en materia de alimentación, con preguntas críticas y reflexivas. Los procedimientos metodológicos utilizados fueron la atención gerontológica a través de la Evaluación Geriátrica Integral (AGA), a partir de las respuestas obtenidas con la AGA, se elaboró un plan de atención individualizado, y acciones de educación alimentaria y nutricional a través de charlas y talleres. Los resultados encontrados fueron de multimorbilidad, alta tasa de enfermedades articulares y osteoporosis, además de Hipertensión Arterial, lo que nos lleva a observar la importancia de la educación alimentaria y nutricional en la contribución para evitar la aceleración de las comorbilidades existentes y en el tratamiento de las deficiencias nutricionales más allá. promover la autonomía alimentaria de esta población.

PALABRAS CLAVE: Envejecimiento. Promoción de la salud. Asesoramiento nutricional. Alimentación saludable.

Recebido em: 01.10.2019. Aceito em: 09.10.2019. Publicado em: 01.01.2020. 


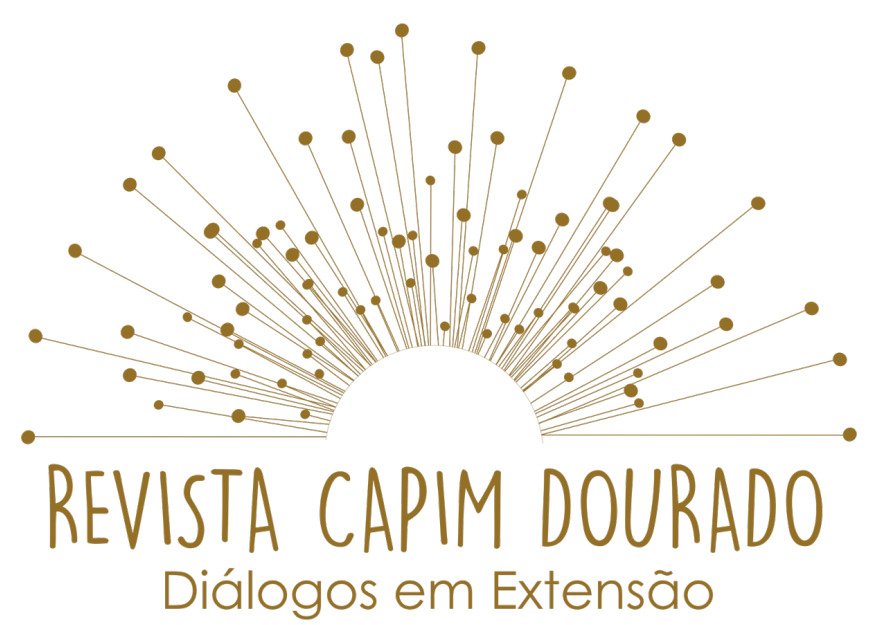

ISSN n² 2595-7341

Vol. 3, n. 1, Janeiro-Abril, 2020

DOI: http://dx.doi.org/10.20873/uft.2595-7341.2020v3n1p41

\section{INTRODUÇÃO}

O envelhecimento da população brasileira é marcado por elevada carga de doenças crônicas não-transmissíveis e incapacidades. $O$ aumento do número de idosos com elevado grau de incapacidades e elevada carga de doenças crônicas é um reflexo do atual perfil demográfico e epidemiológico somado às condições socioeconômicas e de estilo de vida da população brasileira (BRASIL, 2013).

Sabe-se da importância da alimentação adequada para a manutenção das reservas corporais, bem como, para a prevenção e controle de doenças crônicas, porém dados do última Pesquisa de Orçamentos Familiares apontou elevadas prevalências de inadequação na alimentação de idosos; decorrente da pouca variedade de alimentos que compõem a dieta habitual e do consumo insuficiente de frutas, verduras e legumes pelos mesmos, além da elevada ingestão de sódio (FISBERG et al., 2013).

Diante dos indicadores atuais da saúde e nutrição da população idosa e considerando que a Universidade da Maturidade (UMA) é um Programa que tem como objetivo promover qualidade de vida no processo de envelhecimento humano, o objetivo deste projeto foi promover práticas alimentares saudáveis para idosos da UMA/UFT por meio da educação em saúde e da educação alimentar e nutricional.

\section{METODOLOGIA}




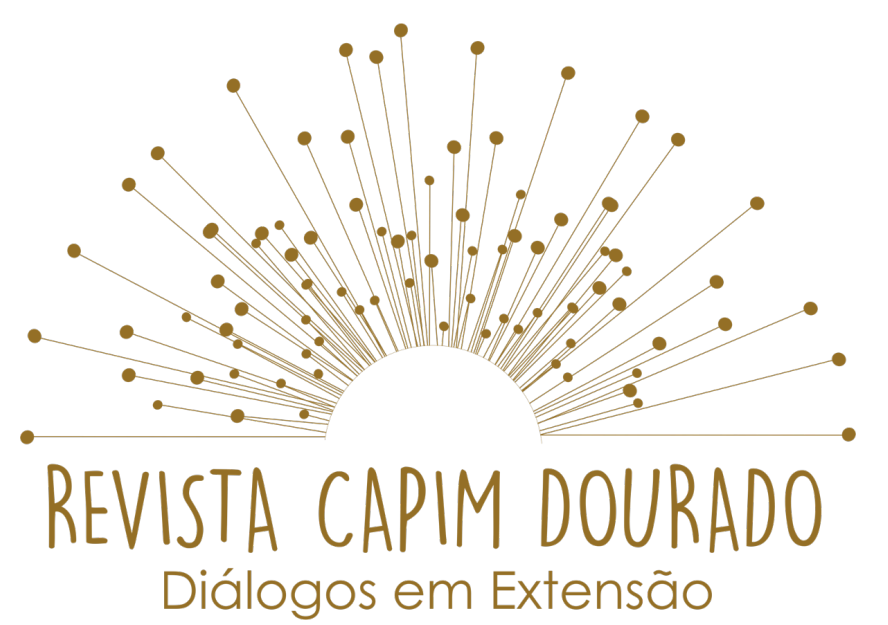

ISSN n² 2595-7341

Vol. 3, n. 1, Janeiro-Abril, 2020

DOI: http://dx.doi.org/10.20873/uft.2595-7341.2020v3n1p41

As atividades foram desenvolvidas de março a setembro de 2018 pela bolsista de extensão sob supervisão docente. Todos os idosos regularmente matriculados na UMA foram convidados a participar das atividades que incluíram: avaliação geriátrica ampla (AGA); educação em saúde a partir do plano de cuidados; educação alimentar e nutricional (EAN) por meio de palestra e oficina.

Para a realização da AGA, os atendimentos foram agendados por telefone conforme interesse e disponibilidade dos idosos e realizados na sala de avaliação geriátrica da UMA/UFT, conduzidos por equipes multidisciplinares, compostas por acadêmicos dos cursos de graduação em nutrição, enfermagem e medicina.

Os planos de cuidados foram individualizados e elaborados a partir dos problemas identificados por meio da AGA. Após a identificação dos problemas, os acadêmicos discutiam o caso entre si e, posteriormente, com o supervisor. Após esta etapa, elaboravam uma cartilha contendo orientações para cada um dos problemas identificados, a qual era revisada pelo docente supervisor. Em consulta de retorno o idoso era esclarecido sobre os problemas, recebia o aconselhamento, as devidas orientações verbais e escritas (cartilha) e o encaminhamento quando necessário.

A ação de EAN se deu por meio de palestra sobre práticas e atitudes alimentares seguida de oficina com exercícios críticos e reflexivos que levantaram questões sobre: representações sociais das doenças crônicas no contexto alimentar e nutricional; como fazer as pazes com a comida; importância do planejamento alimentar; estabelecimento de metas acerca de sua própria 


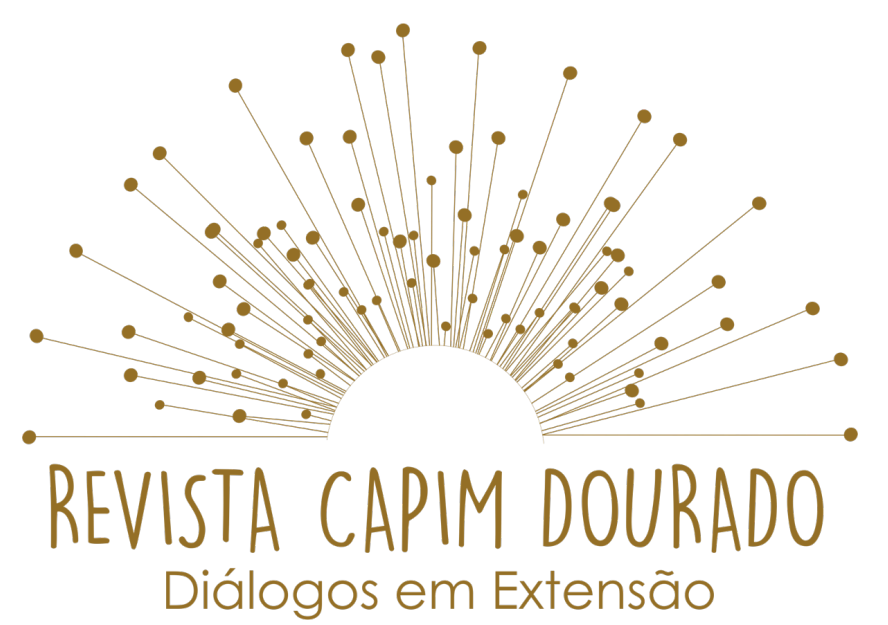

ISSN n² 2595-7341

Vol. 3, n. 1, Janeiro-Abril, 2020

DOI: http://dx.doi.org/10.20873/uft.2595-7341.2020v3n1p41

alimentação dentro de suas limitações. Ao final foram apresentadas receitas culinárias saudáveis como sugestões para a rotina alimentar.

\section{FUNDAMENTAÇÃO TEÓRICA}

De uma população predominantemente jovem em um passado não tão distante, o Brasil segue para o alargamento do topo da pirâmide demográfica, acompanhando a tendência mundial (MIRANDA et al., 2016). As projeções para o ano de 2025 indicam que o contingente de pessoas acima de 60 anos no Brasil será de 34 milhões, com uma expectativa de vida de 75 anos (IBGE, 2010) e o país será o sexto maior do mundo em número de idosos (OMS, 2005).

O envelhecimento é marcado por alterações físicas e funcionais que podem interferir na qualidade de vida do idoso (BUSNELLO, 2007). Neste sentido, é preciso considerar a necessidade de vigilância e monitoramento da saúde da pessoa idosa a fim de intervir precocemente e minimizar os possíveis impactos negativos na saúde dos idosos (BRASIL, 2010).

Com o envelhecimento da população faz se necessárias ações de cuidado, demandando serviços de saúde, junto como avançar da idade aparecem também as doenças e carências nutricionais e para se promover um envelhecimento mais sadio faz se necessárias ações de educação alimentar e nutricional, visando a qualidade de vida e saúde desses idosos.

No contexto da alimentação e nutrição, sabe-se que o estado nutricional pode ser afetado por fatores que modificam as necessidades nutricionais, tais como, doenças, polifarmácia, sedentarismo e também por condições que alteram a 


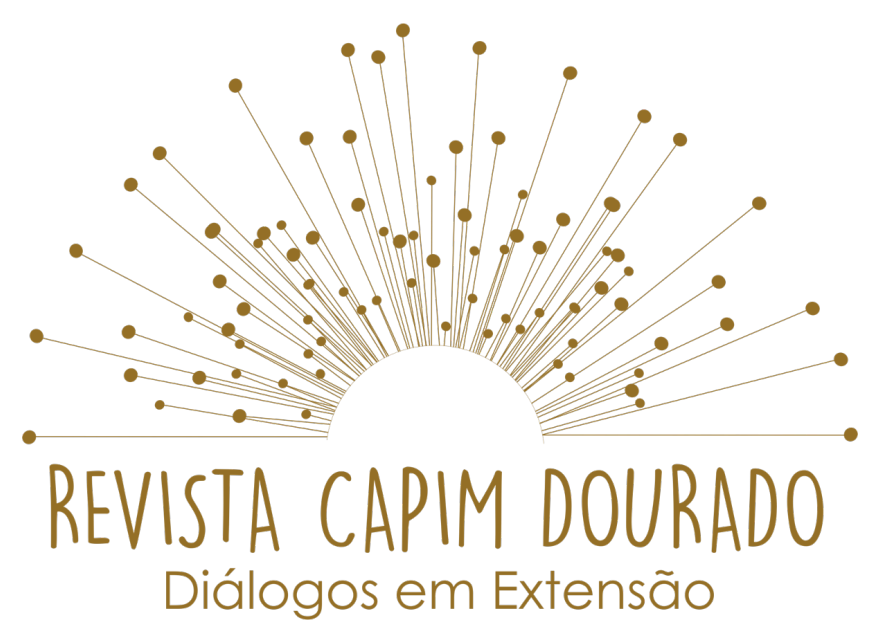

ISSN n² 2595-7341

Vol. 3, n. 1, Janeiro-Abril, 2020

DOI: http://dx.doi.org/10.20873/uft.2595-7341.2020v3n1p41

ingestão alimentar do idoso, tais como, saúde bucal comprometida e doenças, especialmente aquelas que afetam a cognição, coordenação motora, capacidade funcional e humor (DUARTE et al., 2016).

Esse envelhecimento traz consigo a necessidade de uma avaliação mais especifica que leva em consideração não só as doenças mais em todos os aspetos por isso utiliza se para o atendimento gerontológico a AGA, Avaliação Geriátrica Ampla que avalia o idoso através de questionários e obtém um diagnóstico como um todo e não por partes.

A avaliação geriátrica ampla (AGA), instrumento utilizado na avaliação multidimensional de idosos, é capaz de identificar precocemente problemas de saúde e de condições de vida. A AGA tem uma estrutura variável e permite avaliar o perfil socioeconômico, qualidade da saúde, presença de doenças, uso de medicamentos, estado nutricional, rede de apoio social, funcionalidade familiar, fragilidade, capacidade funcional, risco de quedas, violência e maus tratos, entre outros aspectos. A AGA é um instrumento que inclui escalas validadas e seu emprego na prática clínica pode proporcionar inúmeros benefícios à melhoria da qualidade de vida do idoso, pois envolve uma avaliação bastante detalhada com um olhar interdisciplinar (COSTA et al., 2003).

Detectados os problemas dessa fase como podemos agir para que esses problemas possam ser resolvidos? É nesse lugar que entram as ações de educação alimentar e nutricional afim de promover o autocuidado e o autoconhecimento, buscando também a diminuição ou a erradicação de comobirdades associadas a uma alimentação saudável e balanceada. 


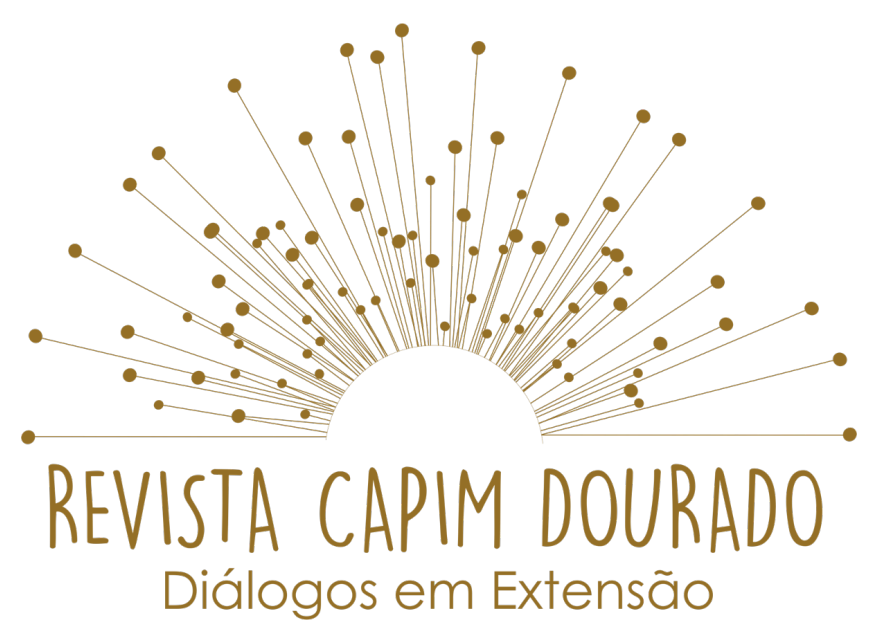

ISSN n² 2595-7341

Vol. 3, n. 1, Janeiro-Abril, 2020

DOI: http://dx.doi.org/10.20873/uft.2595-7341.2020v3n1p41

A Educação Alimentar e Nutricional (EAN) é o campo do conhecimento e de prática contínua e permanente, transdisciplinar, intersetorial e multiprofissional que visa promover a prática autônoma e voluntária de hábitos alimentares saudáveis, contribuindo para assegurar o Direito Humano à Alimentação Adequada (DHAA).

Insere-se em uma das diretrizes da Política Nacional de Segurança Alimentar e Nutricional (PNSAN) e, assim, configura-se como estratégia fundamental para o enfrentamento das problemáticas brasileiras referentes à má nutrição, como o excesso de peso e a obesidade, permitindo refletir sobre toda a cadeia produtiva - produção, abastecimento e acesso a alimentos adequados e saudáveis.

A educação alimentar e nutricional no Brasil está ligada as Políticas de alimentação e nutrição, entendendo um pouco sobre a educação alimentar e nutricional entende se que a EAN, passou por diversas etapas para chegar até a situação atual nas décadas de 1940 a 1970 a EAN estava ligada à fome e principalmente a renda, a partir da década de 80 é que começou a se dar espaço para uma educação alimentar e nutricional mais crítica, levando a alimentação como um direito de todos e a alimentação como parte das estratégias para a promoção da saúde.

\section{RESULTADOS FINAIS}




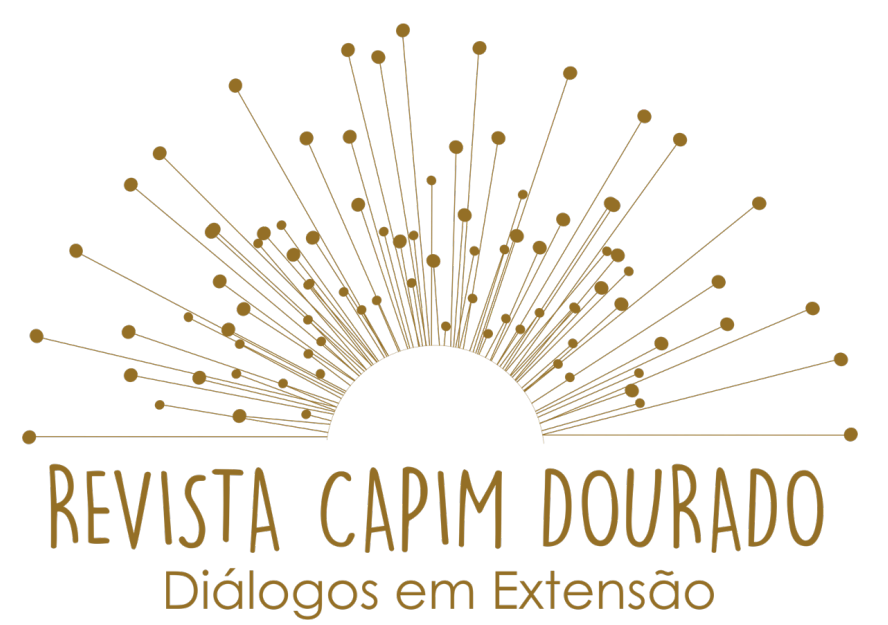

ISSN n² 2595-7341

Vol. 3, n. 1, Janeiro-Abril, 2020

DOI: http://dx.doi.org/10.20873/uft.2595-7341.2020v3n1p41

A AGA foi realizada com 27 idosos, sendo a maioria mulheres (70,4\%), com idade entre 60 e 69 anos (51,9\%), viúva (44,4\%), com renda entre 2 e 4 salários mínimos (48,2\%). A maioria referiu multimorbidade (62,1\%), predominando doenças articulares (59,6\%), osteoporose $(40,7 \%)$ e hipertensão arterial $(37,0 \%)$. A AGA incluiu escalas validadas e seu emprego possibilitou avaliar as diversas dimensões da saúde do idoso exigindo um olhar interdisciplinar (COSTA et al., 2003). Durante os atendimentos realizou-se uma escuta ativa e empática, sendo perceptível nos idosos a necessidade por essa maior atenção durante o diálogo no atendimento, compartilhando problemas familiares, sentimentos e queixas pessoais. A escuta ativa é um método de comunicação importante (MESQUITA e CARVALHO, 2014) que possibilitou uma maior compreensão e entendimento da dimensão dos problemas pessoais de cada idoso.

A elaboração do plano de cuidados individualizado considerou os diferentes contextos sociais, culturais e econômicos dos idosos e contribuiu para a conscientização, orientação e empoderamento dos idosos em relação ao autocuidado com a saúde. A ação de EAN revelou que os diagnósticos de doenças crônicas, tais como, diabetes e hipertensão, representavam para os idosos a privação alimentar e perda do prazer ao comer. Percebeu-se que os mesmos possuíam informações equivocadas acerca da alimentação e doenças crônicas. A oficina buscou conscientizar os idosos sobre a necessidade de resgatar a autonomia alimentar e reforçou a importância do autocuidado e autoconfiança acerca de suas atitudes e práticas alimentares a partir do comer consciente e com atenção plena. 


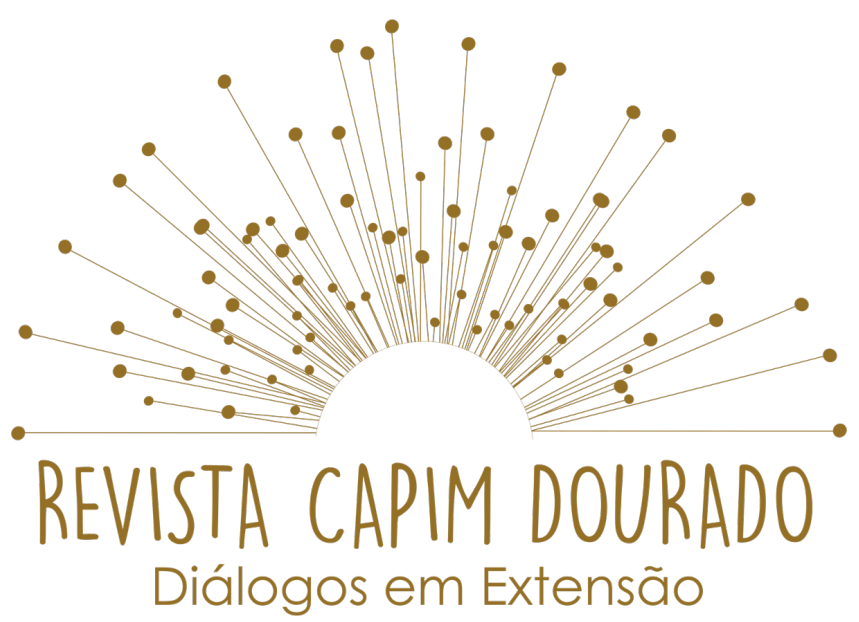

ISSN n² 2595-7341

Vol. 3, n. 1, Janeiro-Abril, 2020

DOI: http://dx.doi.org/10.20873/uft.2595-7341.2020v3n1p41

\section{CONSIDERAÇÕES FINAIS}

Promover educação em saúde e educação alimentar e nutricional para os idosos pode contribuir para evitar complicações de morbidades pré-existentes, prevenir carências nutricionais e melhorar a relação com a comida, contribuindo para autonomia alimentar, autocuidado em saúde e qualidade de vida dos mesmos.

\section{REFERÊNCIAS}

ALMEIDA, C. D. DE; BRANDÃO, B. M. P. PARTICIPAÇÃO E INSERÇÃO SOCIAL: protagonismo da mulher negra em canais do YouTube. Revista Observatório, $v$. 4, n. 1, p. 630-654, 1 jan. 2018.

BARGAS, J.; CAL, D. G. R. LUTA POR RECONHECIMENTO, IDENTIDADES E RELAÇÕES DE PODER: as mulheres no movimento quilombola. Revista Observatório , v. 4, n. 6, p. 475-505, 8 out. 2018.

BRASIL. Ministério da Saúde. Secretaria de Vigilância em Saúde. Departamento de Vigilância de Doenças e Agravos Não Transmissíveis e Promoção de Saúde. Vigitel Brasil 2012: vigilância de fatores de risco e proteção para doenças crônicas por inquérito telefônico. Brasília: Ministério da Saúde; 2013.

BUSNELLO, F. M. Aspectos Nutricionais no Processo do Envelhecimento. São Paulo: Editora Atheneu, 2007. 


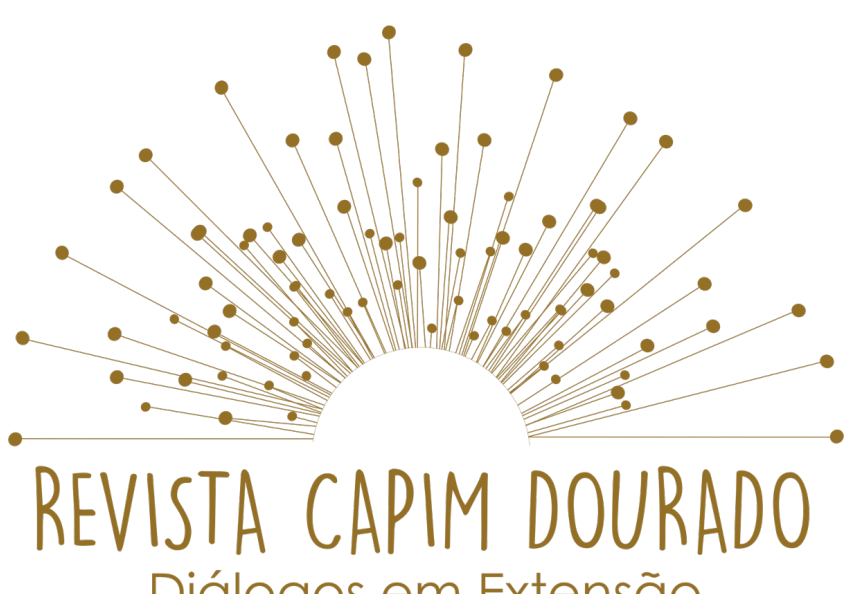

Diálogos em Extensão

ISSN n² 2595-7341

Vol. 3, n. 1, Janeiro-Abril, 2020

DOI: http://dx.doi.org/10.20873/uft.2595-7341.2020v3n1p41

COSTA, E. F. A.; MONEGO, E. T. Avaliação Geriátrica Ampla (AGA). Revista da UFG. Goiânia, v.5, n.2, 2003.

COSTA, Elisa Franco de Assis; MONEGO, Estelamaris Tronco. Avaliação Geriátrica Ampla (AGA). Revista da UFG. Goiânia, v. 5, n. 2, p. 11-15, 2003.

DUARTE, M. S. L.; REZENDE, F. A. C.; SOUZA, E. C. G. Abordagem Nutricional no Envelhecimento. $1^{\text {a }}$ Edição. Rio de Janeiro: Editora Rubio, 2016.

FISBERG, Regina Mara et al. Ingestão inadequada de nutrientes na população de idosos do Brasil: Inquérito Nacional de Alimentação 2008-2009. Rev. Saúde Pública, São Paulo, v. 47, supl. 1, p. 222s-230s, 2013.

IBGE. Instituto Brasileiro de Geografia e Estatística. Censo Demográfico de 2010. [Acesso em 11 de junho de 2018]. Disponível em: $<$ http://www.ibge.gov.br/home/estatistica/populacao/censo2010/>.

GARBI, D. B. L.; SANTOS, K. C. DOS; BARRETO, L. K. DA S.; GARBI, G. P. POR QUE O EMPODERAMENTO DAS MULHERES NEGRAS CAUSA MEDO?. Revista Observatório , v. 5, n. 6, p. 954-958, 1 out. 2019.

GONÇALVES MAIA, M. F.; TRINDADE ROCHA, J. D.; MERIQUI RODRIGUES, M. MÍDIAS EDUCATIVAS "MAIS MULHERES": um centro de documentação interdisciplinar de gênero e comunicação em Palmas, Tocantins, Brasil. Revista Observatório , v. 3, n. 4, p. 427-444, 1 jul. 2017.

MESQUITA, Ana Cláudia; DE CARVALHO, Emília Campos. A. Escuta Terapêutica como estratégia de intervenção em saúde: uma revisão integrativa. Rev. esc. enferm. USP, São Paulo, v. 48, n. 6, p. 1127-1136, 2014. 


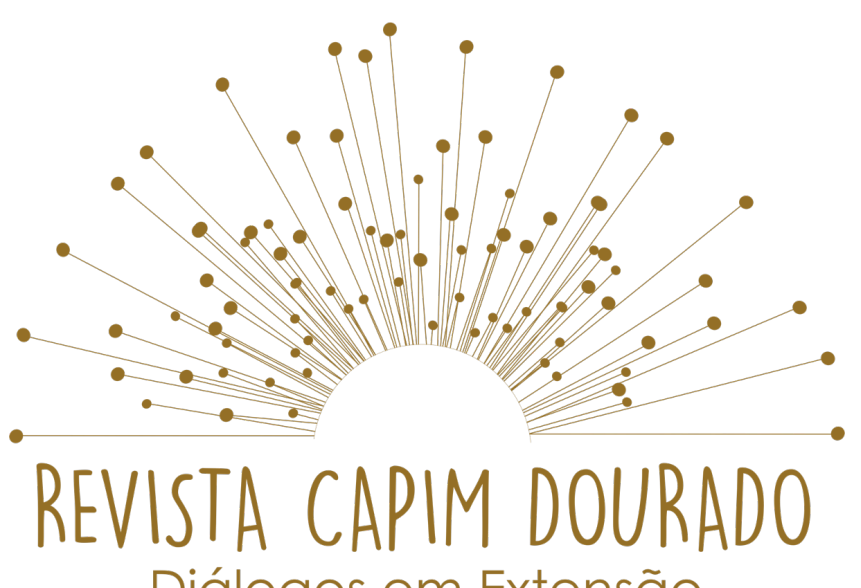

Diálogos em Extensão

ISSN n² 2595-7341

Vol. 3, n. 1, Janeiro-Abril, 2020

DOI: http://dx.doi.org/10.20873/uft.2595-7341.2020v3n1p41

MANSO, M. E. G.; MELLO, R. G. R. DE; LOPES, R. G. DA C. MÍDIAS DIGITAIS E AS INVISÍVEIS VIOLÊNCIAS CONTRA IDOSOS. Revista Observatório , v. 4, n. 2, p. 265278, 1 abr. 2018.

MIRANDA, G. M. D.; MENDES, A. C. G.; SILVA, A. L. A. O envelhecimento populacional brasileiro: desafios e consequências sociais atuais e futuras. Revista Brasileira de Geriatria e Gerontologia, Rio de Janeiro, v.19, n.3, p.507-519, 2016. MIRANDA, C. G. L.; CASTRO, M. S.; SOARES-SOBRINHO, J. L. CONSTRUÇÃO COMPARTILHADA DE VÍDEO PARA EDUCAÇÃO DE IDOSOS HIPERTENSOS. Revista Observatório , v. 5, n. 6, p. 933-948, 1 out. 2019.

ORGANIZAÇÃO MUNDIAL DA SAÚDE. Envelhecimento ativo: uma política de saúde. Brasília: Organização Pan-Americana de Saúde; 2005.

Princípios e Praticas para Educação Alimentar e Nutricional, Caderno EAN, Revista do CFN. Brasilia,2018.

SILVA NETO, L. S.; MACEDO, M. DE L. L.; OSÓRIO, N. B.; SECHIM, W. Z.; SANTOS, J. S. DOS. NARRATIVAS DE MULHERES: as perdas e o luto. Revista Observatório, v. 4, n. 6, p. 776-793, 8 out. 2018. 